\title{
La garantía de los derechos de la mujer en el ordenamiento jurídico colombiano
}

\author{
Jimena Cardona Cuervo* \\ Yudy Andrea Carrilo Cruz ${ }^{* *}$ \\ Rosa María Caycedo Guió
}

Resumen

\begin{abstract}
Recibido: 12 de noviembre del 2018
Evaluado: 24 de noviembre de 2018

Aceptado: 28 de noviembre del 2018

Citar como: Cardona Cuervo, J., Carrillo Cruz, Y. A. y Caycedo Guió, R. M. (2019). La garantía de los derechos de la mujer en el ordenamiento jurídico colombiano. Hallazgos, 16(32), 83-106. DOl: https://doi. org/10.15332/2422409X.3265
\end{abstract}

En este trabajo se pretende analizar la efectividad del compendio normativo colombiano en la garantía de los derechos de las mujeres, haciendo énfasis en las medidas de atención que se han diseñado para que la mujer pueda salvaguardarse de la violencia y emprender su proyecto de vida. Se fundamenta en una metodología cualitativa con enfoque explicativo basada en el método bibliográfico, es decir, en una revisión sistemática y crítica de la normatividad nacional sobre la protección de la mujer, contrastada con hechos reales. Las conclusiones a las que se llega permiten inferir que con el desarrollo normativo internacional de la salvaguarda de la mujer, se generó una revolución jurídica en Colombia que culminó con un sólido compendio de instrumentos a favor de la mujer pero que no se ha cerrado la brecha entre el ser y el deber ser, lo que deja a la mujer sin protección en los escenarios de violencia.

Palabras clave: derechos humanos, igualdad, mujer, protección, violencia.

Abogada, magíster em Ciência Jurídica de la Universidade Do Vale Do Itajaí, Brasil (2017), magíster en Derecho Público de la Universidad de Caldas (2016), especialista en Derecho Constitucional (2012) y en Derecho Administrativo (2011) de la Universidad Santiago de Cali. Asesora, litigante y miembro del Nodo de Innovación Social de la Universidad Cooperativa de Colombia, docente de la misma institución e investigadora en diversos proyectos académicos. Bogotá D. C., Colombia. Correo electrónico: jimena.cardona@campusucc.edu.co - ORCID: https://orcid. org/0000-0001-9850-2321

** Abogada, magíster en Derecho Administrativo de la Universidad Libre (2016), especialista en Docencia Universitaria de la Universidad Cooperativa de Colombia (2013), especialista en Derecho Constitucional y Parlamentario de la Universidad Externado de Colombia (2008), líder del grupo de investigación Abogados Solidarios de la Universidad Cooperativa de Colombia, docente de la misma institución e investigadora en diversos proyectos académicos. Bogotá D. C., Colombia. Correo electrónico: yudy.carrillo@campusucc.edu.co

*** Abogada, especialista en Alta Gerencia y Economía Solidaria (2009), especialista en Docencia Universitaria de la Universidad Cooperativa de Colombia (2013). Miembro del grupo de investigación Abogados Solidarios de la Universidad Cooperativa de Colombia, docente de la misma institución e investigadora en diversos proyectos académicos. Bogotá D. C., Colombia. Correo electrónico: rosacaycedo@campusucc.edu.co - ORCID: https:// orcid.org/0000-0002-7180-1855 


\section{Guarantee of women's rights in the Colombian legal system}

\begin{abstract}
This work intends to analyze the effectiveness of the Colombian normative compilation in the guarantee of women's rights, emphasizing the protection measures that have been designed so women can safeguard themselves from violence and undertake their life project. It is based on a qualitative methodology with an explanatory approach based on the bibliographic method that is, on a systematic and critical review of the national regulations on the protection of women, contrasted with real events. The conclusions reached allow us to infer that with the international normative development of women's protection, a legal revolution was generated in Colombia that culminated in a solid compilation of instruments in favor of women but that the gap between women and men, what is and what ought to be, has not been closed, leaving women without protection in the scenarios of violence.
\end{abstract}

Keywords: Human rights, equality, woman, protection, violence.
Received: november 12, 2018

Evaluated: november 24, 2018

Accepted: november 28, 2018 


\section{A garantia dos direitos da mulher no ordenamento jurídico colombiano}

Recebido: 12 de novembro de 2018

Avaliado: 24 de novembro de 2018

Aceito: 28 de novembro de 2018

\section{RESUMO}

Neste trabalho pretende-se analisar a efetividade do compendio normativo colombiano na garantia dos direitos das mulheres, enfatizando nas medidas de atenção que se tem desenhado para que a mulher possa se salvaguardar da violência e empreender seu projeto de vida. Fundamenta-se em uma metodologia qualitativa com um enfoque explicativo baseado no método bibliográfico, aliás, em uma revisão sistemática e crítica da normatividade nacional sobre a proteção da mulher, contrastada com fatos reais. As conclusões as quais se chega permitem inferir que com o desenvolvimento normativo internacional da salvaguarda da mulher, gerou-se uma revolução jurídica na Colômbia que terminou com um sólido compêndio de instrumentos a favor da mulher, mas que não tem se fechado a brecha entre o ser e o dever ser, o que deixa a mulher sem proteção nos cenários da violência.

Palavras-chave: diretos humanos, igualdade, mulher, proteção, violência. 


\section{INTRODUCCIÓN}

La justicia de género ${ }^{1}$ y su aplicación práctica, son fundamentales para superar los obstáculos que enfrentan las mujeres en la búsqueda de la plena realización de sus derechos humanos. No puede negarse que en Colombia se han producido importantes reformas legales y reglamentarias desde el reconocimiento de su ciudadanía en 1954 y pasando por la Constitución Política de 1991, sin perder de vista el contexto global en el que se desenvuelve el país. Sin embargo, en medio de un ordenamiento jurídico garantista de los derechos de la mujer, persisten altos índices de violencia en su contra y estas deben superar serios obstáculos para acceder a la justicia y lograr reparación, lo que evidencia una brecha entre el "deber ser" y el "ser". Por ello, en este trabajo se pretende abordar el tema del derecho como herramienta imprescindible en la necesidad de limitar toda práctica que desconozca y vulnere la dignidad de las mujeres en Colombia, a partir de los esfuerzos legislativos y gubernamentales para dotar de instrumentos jurídicos en defensa de los derechos de aquellas, inspiradas en la Declaración de Derechos Humanos y documentos análogos, que establecen los principios de igualdad y no discriminación. El examen de la normatividad y los programas que en Colombia se han desarrollado con el fin de reconocer, fomentar y proteger los derechos de la mujer es indispensable para apreciar los avances en la defensa de estos derechos en un contexto colmado de agresiones, en el que ellas son

"Cuando se habla de justicia de género debemos saber que esta no busca una justicia particular para las mujeres en tanto tales, sino que los parámetros a través de los cuales se comprende y se aplica justicia, no refuercen ni reproduzcan las discriminaciones que hacen parte del sistema sexo/género" (Facio, A. y Fries, 1999). las principales víctimas de violencias relacionadas con el género, tanto en el espacio público como en el privado, a pesar de importantes reformas constitucionales, legales y reglamentarias adelantadas en el país.

Para cumplir con el propósito de este artículo, se realizará un recuento histórico de la protección a la mujer en el ámbito nacional haciendo especial énfasis en la Ley 1257 del 2008 y en las medidas de protección adoptadas en favor de las mujeres víctimas de violencia de género, con miras a adelantar un análisis crítico de los mecanismos diseñados en el país para hacerle frente a la violencia de género y garantizar la protección de las mujeres.

Este trabajo es de corte cualitativo y enfoque exploratorio y su intención es explicar los fenómenos encontrados vinculados a la brecha entre el sólido compendio normativo en favor de la mujer colombiana y la deficiencia de su protección en el terreno de la práctica. El método aplicado para alcanzar el propósito anunciado se basó en la revisión selectiva de literatura, de conformidad con lo esbozado por Hernández, Fernández, y Baptista (2008) quienes consideran al examen documental como la principal herramienta de acceso a la informacion en trabajos cualitativos.

\section{LOS PRIMEROS DERECHOS DE LA Mujer en Colombia}

Importantes instrumentos internacionales en el ámbito mundial como la Declaración de los Derechos del Hombre y del Ciudadano (1789) y la Declaración Universal de los Derechos Humanos (1948) ignoraron a la mujer como un sujeto de derecho, pero a raíz 
de su lucha por conquistar un nuevo estatus, distintos organismos supranacionales se encargaron de reivindicarlo y propender la modificación progresiva de los sistemas legales para dar a la mujer un nuevo lugar en la sociedad.

Con la Convención sobre los Derechos Políticos de la Mujer (1952), la Convención sobre la Nacionalidad de la Mujer Casada (1957), la Convención Sobre la Eliminación de Todas las Formas de Discriminación Contra la $\mathrm{Mu}$ jer (1979), la Convención de Belén do Pará de (1994) y el Programa Interamericano sobre la Promoción de los Derechos Humanos de la Mujer y la Equidad e Igualdad de Género (2000), se abrió un importante espacio de deliberación y compromisos para la protección y defensa de los derechos de la mujer, lo que dio como resultado la transformación sustantiva de la normatividad colombiana, no sin arduas controversias. En palabras de Blanco y Cárdenas (2009): “Los derechos de las mujeres no se planearon históricamente, no se otorgaron por decisión política. Estos son el resultado de la lucha desmedida de las mujeres por el bienestar de los suyos, como lo fue en un principio" (p. 156).

Tal como lo consagraban legislaciones latinoamericanas, la mujer, una vez contraía matrimonio, se asimilaba a un menor de edad o a un demente: no podía administrar libremente sus bienes y carecía de capacidad civil. En consecuencia, le estaba prohibido celebrar contratos, comparecer a un estrado judicial, hacer compras de bienes suntuosos, entre otros actos jurídicos, para los cuales requería la autorización del esposo, el cual ejercía la potestad patrimonial².

2 Código Civil Colombiano (CCC). Ley 57 de 1887, art. 170, abril 15 de 1887 (Colombia): "La potestad patrimonial es el conjunto de
Esta situación de desigualdad y opresión de la mujer generó movimientos feministas que propendían el reconocimiento de su capacidad jurídica. La acción de estos grupos se sumó a la disposición gubernamental de equilibrar los derechos y obligaciones en el matrimonio, haciendo eco a los avances de otros países. A este respecto, Gómez (2015) afirma que:

Varios miembros del Gobierno del presidente Olaya Herrera argumentaban que países como Suecia, Finlandia, Noruega y Dinamarca eran parte de un movimiento universal que perseguía la emancipación económica de la mujer y, por ello, eran sociedades más avanzadas que la colombiana. (P. 69).

Con este fundamento, entre otras manifestaciones de capacidad intelectual de la mujer que contradecían las tesis de inferioridad, se aprobó la Ley 28 de 1932, la primera norma colombiana en reivindicar los derechos de la mujer. En virtud de lo dispuesto en ella se llevó a cabo una reforma al régimen patrimonial del matrimonio, otorgándole a la mujer la plena administración de los bienes. Dispuso la ley que "la mujer casada, mayor de edad, como tal, puede comparecer libremente en juicio, y para la administración y disposición de sus bienes no necesita autorización marital ni licencia del juez, ni tampoco el marido será su representante legal" (Ley 28 de 1932).

Habiendo logrado una gran conquista, la mujer continuó su lucha para obtener el reconocimiento de otros derechos como los de orden político, obteniendo que en

derechos que las leyes conceden al marido sobre la personas y bienes de la mujer". 
la reforma constitucional de 1936 se reconociera el derecho de las mujeres a ocupar empleos en la administración pública. Todavía se veía lejana la posibilidad de ejercer el derecho al sufragio (Acto Legislativo 03 de 1954.).

Posteriormente, con el reconocimiento del derecho al voto para las ciudadanas en varios países, los movimientos feministas se apresuraron a ejercer presión para modificar su condición jurídica. Colombia no fue la excepción a esta tendencia, aunque con marcada resistencia y oposición por parte de la Iglesia católica que condujo al archivo varias iniciativas de voto femenino hasta 1954 cuando, mediante el Acto Legislativo número 03, se reconoció el derecho de la mujer a elegir y a ser elegida. Respecto a la influencia de la religión como obstáculo a los avances de los derechos de las mujeres, Blanco y Cárdenas (2009) afirman que:

La Iglesia, más allá de lo religioso, marcó la línea de comportamiento para la mujer, de absoluta estigmatización, subvaloración y exclusión (p. 145)... El problema de los derechos de las mujeres en Colombia es un tema que está sujeto a un elemento real que aún pervive y que le ha obstaculizado gran parte de su realización individual, familiar y profesional, pero que le ha definido casi todos los logros alcanzados, es decir, el tema de la religión. (P. 155).

Si bien es cierto que la mujer obtuvo derechos civiles en medio de grandes dificultades, fue la Constitución de 1991 la que le abrió el paso a la igualdad formal y material al consagrar derechos de rango fundamental que pretenden garantizar que todas las personas sean tratadas con dignidad.
El recorrido por la Carta Magna permite constatar la existencia de disposiciones introducidas exclusivamente para eliminar la marginalidad femenina como la garantía de participación en los altos niveles de la administración (artículo 40), la igualdad de derechos y oportunidades del hombre y la mujer (artículo 43) y la indicación al Congreso que debe incluir la protección a la mujer en el estatuto de trabajo (artículo 53). Dichas disposiciones conforman un robusto compendio de garantías que sirvieron de base para diseñar el actual sistema normativo el cual tiene como objetivo proteger los derechos de la mujer y salvaguardarla de la violencia.

El nuevo marco constitucional dio pasos ciertos que, aunque no suficientes, coadyuvan con el posicionamiento de las mujeres como sujetos de derechos y partícipes en la construcción del Estado. Hoy existe una consciencia un tanto mayor de la necesidad de proteger a la mujer de la discriminación, así como de la premura de concederle una protección especial en ciertos espacios y en ciertas circunstancias. (Acosta, 2007, p. 53).

A pesar de la existencia de una carta de navegación colmada de principios y derechos, que además encomienda al Congreso de la República y al Gobierno la promulgación de normas y políticas públicas, la mujer sigue necesitando escenarios de participación y respeto. En este sentido, se inició la protección de la mujer cuando se encuentra en situación de vulnerabilidad, especialmente cuando asume solitariamente las cargas de la familia.

En 1993, con la Ley 82 se creó un estatuto de garantías para la mujer cabeza de familia o jefe de hogar que incluye oportunidades 
de educación, empleo y vivienda, así como prelaciones para sus hijos a la hora de acceder a la educación o requerir material didáctico.

En esta norma se define claramente el concepto de jefatura femenina del hogar sin circunscribirlo a la crianza de los hijos o a la ausencia de pareja, contrario al imaginario social del jefe de familia como una mujer sin convivencia marital, esencialmente madre. Es importante resaltar que la ley contempla a una mujer casada como cabeza de familia, cuando su esposo se encuentra en una situación que le impide aportar a la construcción de la familia o simplemente está ausente. Igual calidad le otorga a la mujer que tiene a su cargo menores de edad o discapacitados que no necesariamente deben ser sus hijos. De hecho, también es jefe del hogar cuando es la directora de la casa, sin que los demás miembros de la familia le apoyen en los menesteres.

Mujer cabeza de familia, quien, siendo soltera o casada, ejerce la jefatura femenina de hogar y tiene bajo su cargo, afectiva, económica o socialmente, en forma permanente, hijos menores propios $\mathrm{u}$ otras personas incapaces $\mathrm{o}$ incapacitadas para trabajar, ya sea por ausencia permanente $\mathrm{o}$ incapacidad física, sensorial, síquica o moral del cónyuge o compañero permanente o deficiencia sustancial de ayuda de los demás miembros del núcleo familiar. (Ley 82 de 1993 reformada por la Ley 1232 del 2008).

La especial protección de la mujer cabeza de familia se reflejó en la obligación del Gobierno de proporcionarle condiciones de mayor favorabilidad para equipararla en sus derechos; por ello se establecieron programas particulares para las mujeres cabeza de familia como la instrucción en el Sena, el apoyo a organizaciones sociales de vivienda y desde la banca el diseño de una línea de crédito especial para que logre acceder fácilmente a unidades de negocio ${ }^{3}$.

Con relación a la participación femenina en los altos cargos gubernamentales, solo se contaba con la mera enunciación constitucional y ante la falta de reglamentación legal los nominadores contrataban principalmente a hombres. De allí que en el año 2000 se expidiera la Ley 581, conocida como la ley de cuotas, mediante la cual se estipuló que a la mujer debe asegurársele la participación en los cargos públicos en un mínimo del $30 \%$ incluyendo, además de los altos niveles, aquellos cargos provistos en provisionalidad y de libre nombramiento y remoción, tanto en el ámbito nacional como en el local.

La clase política androcéntrica se resistía; sostenía que la mujer en Colombia no había nacido para gobernar la cosa pública y hacer política, considerando que su obrar en la sociedad era realizado por medios indirectos, entre ellos, gobernar el hogar y contribuir a la generación de costumbres delicadas, suaves y profundas (Montoya, 2009, p. 143).

3 "El artículo $3^{\circ}$ de la Ley 82 de 1993 quedará así: Artículo 3․ Especial protección. El Gobierno nacional establecerá mecanismos eficaces para dar protección especial a la mujer cabeza de familia, promoviendo el fortalecimiento de sus derechos económicos, sociales y culturales, procurando establecer condiciones de vida dignas, promoviendo la equidad y la participación social con el propósito de ampliar la cobertura de atención en salud y salud sexual y reproductiva; el acceso a servicios de bienestar, de vivienda, de acceso a la educación básica, media y superior incrementando su cobertura, calidad y pertinencia; de acceso a la ciencia y la tecnología, a líneas especiales de crédito y a trabajos dignos y estables". (Ley 1232, 2008). 
Continuando con la protección de las mujeres, especialmente de aquellas que se encuentran en situación de vulnerabilidad, en 2002 se promulgó la Ley 731 con el fin de mejorar la calidad de vida de la mujer rural y diseñar estrategias que le permitan ser productiva y progresar según su propio plan de existencia.

La mujer que trabaja en el campo, ya sea como prestadora de servicios, labradora de la tierra o en el mercadeo de productos agrícolas, tiene derecho a participar del fondo de fomento para la mujer rural que dispone de apoyo económico a procesos productivos, es decir, que facilita recursos a la mujer para que promueva su crecimiento familiar, con tasas y plazos accesibles. Así mismo, en la adjudicación de predios rurales, producto de las reformas agrarias, se deberá tener en cuenta a las mujeres, asegurándose de que en cabeza de ellas quede un porcentaje considerable de las tierras. En los proyectos de reforestación se deberá emplear como mínimo un $30 \%$ de las mujeres rurales y las entidades territoriales deberán incluir en sus planes de desarrollo los lineamientos necesarios para que la mujer rural tenga participación y oportunidades.

Con base en esta legislación, el Ministerio de Agricultura lanzó el programa "Mujer Rural" encaminado a fomentar proyectos productivos, del cual, según el Ministerio (2015) "se han beneficiado 11181 mujeres campesinas, con una inversión de \$17 700 millones". El programa cubre desde la capacitación en derechos humanos y en trabajo del campo, hasta la promoción de la propiedad asociativa de la tierra.

El objetivo del programa de Mujer Rural es contribuir al mejoramiento de las condiciones de vida de las mujeres en el campo y la disminución de su situación de pobreza y vulnerabilidad, mediante el incremento del ingreso y el desarrollo de capacidades personales, empresariales y asociativas. (Ministerio de Agricultura, 2015).

En consonancia con el principio de igualdad y no discriminación, en 2003 se expidió la Ley 823 con el objetivo de igualar las oportunidades de la mujer en aspectos sensibles de la vida, como la educación y el trabajo, no solo en lo público, sino también en el escenario privado.

Un aspecto para resaltar en la norma es el diseño de programas a cargo del Gobierno, los cuales deben orientarse a la erradicación de estereotipos sexistas que asocian a la mujer con determinados desempeños laborales y la demeritan a la hora de explotar toda su capacidad. Para ello, se prevé el estímulo de investigaciones sobre género e igualdad de la mujer, disponiendo del presupuesto necesario para realizarlos y con base en ellos adelantar políticas públicas que culminen con la igualdad real de la mujer.

Pese a que la Ley 823 del 2003 consagró un trabajo importante a cargo del Gobierno nacional, que incluía proyectos de capacitación en derechos humanos y en el desempeño de oficios para las mujeres, al igual que acciones que fomentan la capacitación en salud reproductiva, trabajo y demás aspectos sensibles del desarrollo de la mujer, no contempló medidas para su protección en casos de violencia ni procedimientos para disminuir los índices de maltrato, tal como se ha enunciado desde el acogimiento de instrumentos internacionales sobre derechos humanos de la mujer y así lo reconoce la Procuraduría General de la Nación (2004): 
El Estado es responsable de otorgar esa respuesta jurídica particular, para que las mujeres puedan ejercer en debida forma esos derechos, que además están reconocidos en la propia convención contra la discriminación de la mujer. Ordena a los Estados a que tomen todas las medidas necesarias para erradicar todas las formas de discriminación de la mujer... (P. 161).

\section{INSTRUMENTOS LEGALES PARA LA PROTECCIÓN EFECTIVA DE LOS DERECHOS DE LA MUJER}

Aunque el reconocimiento de los derechos de la mujer era visible incluso desde antes de la Carta Magna de 1991, estas carecían de un compendio normativo que las dotara de herramientas para salvaguardarse de sus agresores y vivir en paz; por ello, luego de discutir en el Congreso la necesidad de proteger integralmente a las mujeres, se consolidó en 2008 la Ley 1257, con el fin de sensibilizar, prevenir y sancionar las formas de violencia y discriminación contra las mujeres ${ }^{4}$.

Por primera vez en el desarrollo normativo colombiano en favor de la mujer, se establecieron en forma contundente unos lineamentos que van más allá de la enunciación de derechos, propendiendo por su garantía y haciendo énfasis en la erradicación de la violencia contra la mujer. Estas herramientas, que serán descritas en el desarrollo de este trabajo, están reforzadas por un equipo de

4 El suministro de la información requerida para formular los planes y estrategias que lleven al cumplimiento de esta ley fue encargada a la Consejería Presidencial para la Equidad de la Mujer, a través del Observatorio de Asuntos de Género, entidades que, de la mano de la Mesa Interinstitucional para Erradicar la Violencia contra las Mujeres, han contribuido al fortalecimiento de las medidas de protección de la mujer. trabajo creado por el Decreto 164 del $2010^{5}$ y conformado entre entidades del sector público de carácter especializado y del más alto nivel gubernamental. En esta "mesa interinstitucional", como es llamado el equipo, tienen asiento desde el ministro del Interior hasta el director del Instituto de Bienestar Familiar. La mesa tiene como misión, entre otros aspectos, apoyar el diseño, implementación y evaluación de políticas públicas para lograr el acceso de las mujeres a los servicios y el cumplimiento real de sus derechos. Su trabajo se evidencia especialmente en los aportes realizados para la adecuada reglamentación de la Ley 1257 del 2008.

Concretamente, esta ley define el concepto de violencia contra la mujer y hace referencia a las herramientas que tiene la mujer para ejercer sus derechos y defenderse de agresiones.

Por violencia contra la mujer se entiende cualquier acción u omisión que le cause muerte, daño o sufrimiento físico, sexual, psicológico, económico o patrimonial por su condición de mujer, así como las amenazas de tales actos, la coacción o la privación arbitraria de la libertad, bien sea que se presente en el ámbito público o en el privado. (Ley 1257, 2008).

Con base en este concepto de violencia, la mujer es víctima de maltrato aunque los hechos no comporten agresión física ni se cometan actos violentos, toda vez que la

5 "Artículo 1". OBJETO. El presente decreto tiene por objeto crear la Comisión Intersectorial denominada "Mesa Interinstitucional para Erradicar la Violencia contra las Mujeres", cuyo propósito es aunar esfuerzos para la articulación, coordinación y cooperación entre las entidades, a fin de lograr la atención integral, diferenciada, accesible y de calidad a las mujeres víctimas de la violencia, para lo cual determinará las pautas de su funcionamiento". (Decreto 164, 2010). 
amenaza de daño se configuró como una forma de violencia en sí misma, dada la intimidación que se ejerce contra la mujer sometiéndola a un constante temor ante la inminente configuración de la amenaza de golpes, abuso sexual o violencia económica.

La tesis de violencia contenida en esta norma se extendió a otras formas de maltrato; el concepto de daño derivado de los casos de violencia también se refirió a otras consecuencias que no necesariamente son evidentes, como los golpes, moretones, inflamaciones y otras secuelas de violencia física, lo que generaría específicamente el daño o sufrimiento físico.

La norma establece una tipología de daños, empezando por describir el daño psicológico, como aquel que proviene de la humillación, aislamiento, manipulación, amenaza o degradación de la mujer. El daño o sufrimiento sexual como las consecuencias de constreñir a la mujer para sostener relaciones sexuales o contacto sexualizado verbal o físico, por cualquier medio que prive o límite el consentimiento. También se establece el daño patrimonial como consecuencia de la sustracción, inutilización o retención de los bienes, documentos, títulos valores y demás pertenencias de la mujer.

En materia de derechos, la norma en cuestión enunció los derechos de la mujer y enlistó los derechos de las víctimas de la violencia de género, adicionales a los derechos que para las víctimas ha consagrado el estatuto penal colombiano y las normas específicas sobre violencia sexual. Esto con el fin de ampliar el espectro de sus derechos y garantías, reforzando su protección con la indicación de que todos los derechos de la mujer son derechos humanos.
En términos de Rodríguez y Rodríguez (2014), es necesario promover en la población colombiana la importancia de la mujer como persona digna de respeto y consideración, lo que conllevará a mejorar las relaciones y el tejido social, para que ellas puedan expresar sus potencialidades en beneficio de la sociedad (p. 82). Por ello, a través del Decreto 4798 del $2011^{6}$ el legislador adoptó una serie de medidas destinadas a sensibilizar y prevenir la violencia de género en virtud de las cuales el Gobierno nacional, a través del Ministerio de Educación, debe capacitar a la comunidad en el respeto de los derechos de la mujer como un componente de la cátedra de derechos humanos. Igualmente, debe sensibilizar a funcionarios de las ramas del poder público para que conozcan las formas de violencia contra la mujer y contribuyan a la garantía de sus derechos.

En el aspecto laboral de las mujeres, con el Decreto 4463 del $2011^{7}$ el Ministerio de la Protección Social tiene a su cargo la verificación del cumplimiento de las normas internacionales sobre no discriminación e igualdad en el trabajo. Debe implementar los mecanismos necesarios para que el salario de la mujer corresponda a su esfuerzo y no sea inferior al del hombre, bajo el principio de "igual trabajo, igual salario". También está encargado de garantizar que

6 Este decreto tiene por objeto reglamentar parcialmente la Ley 1257 del 2008. Por medio de este decreto se dictan normas sobre los derechos humanos de las niñas, adolescentes y las mujeres en el ámbito educativo, haciendo énfasis en la implementación de proyectos pedagógicos por parte de las instituciones de educación tendientes a que las mujeres vivan libres de violencias.

7 "Artículo 1. Objeto. El presente decreto tiene por objeto definir las acciones necesarias para promover el reconocimiento social y económico del trabajo de las mujeres, implementar mecanismos para hacer efectivo el derecho a la igualdad salarial y desarrollar campañas de erradicación de todo acto de discriminación y violencia contra las mujeres en el ámbito laboral". (Decreto 4463, 2011). 
las denuncias por acoso laboral y sexual presentadas por mujeres contra sus empleadores sean debidamente atendidas y resueltas, protegiendo a la mujer del despido injusto como consecuencia de las denuncias de violencia.

En lo que respecta a la salud, la Ley 1257 2008 encomendó al Gobierno la realización de protocolos de atención y prevención de la violencia contra la mujer, al igual que la destinación de recursos para la puesta en marcha de un programa asistencial que brinde cuidados, resguardo y recuperación tanto a las mujeres violentadas como a sus hijos, para que, en caso de violencia intrafamiliar, no se vean obligadas a cohabitar con el agresor. La asistencia concedida a las mujeres se denominó medidas de protección y de atención, siendo esta última la que se abordará con especial cuidado en este trabajo, ya que reviste características novedosas y se erige como una poderosa herramienta de defensa de los derechos de la mujer.

Estas medidas enunciadas someramente por la citada ley pretenden salvaguardar los derechos de las mujeres víctimas y de sus hijos, principalmente manteniéndolos alejados del agresor y son especialmente efectivas cuando el victimario es el compañero permanente o esposo, con quien cohabita la mujer. En este punto, es esencial resaltar que el entorno familiar es un escenario peligroso para las mujeres colombianas. La Presidencia de la República, por conducto de la Consejería para la Equidad de la Mujer (2016), afirmó que:

El entorno familiar es un lugar de riesgo para las mujeres colombianas. Esta es una de las conclusiones frente al informe presentado por el Instituto
Nacional de Medicina Legal, Forensis 2015 datos para la vida. Las estadísticas entregadas por esta entidad, señalan como el principal homicida de las mujeres a su pareja o expareja, con 114 casos en 2015, cifra que mostró un descenso en comparación con el 2014 donde se presentaron 145 casos, pero que no deja de ser un panorama preocupante: una mujer asesinada cada 3 días por su pareja o expareja.

En igual sentido, el Instituto de Medicina Legal y Ciencias Forenses (2016) afirma que:

La vivienda es claramente el lugar donde más hechos de violencia sexual se presentan, para el 2014 los casos ocurridos allí representaron el $71 \%$ del total de casos, para el 2015 el $71.8 \%$, mientras que para el 2016 representaron el $72.5 \%$ (p. 19). Los familiares aparecen como los principales presuntos responsables de los casos de violencia sexual, para 2014 se registran 4.696 casos en los cuales el presunto agresor es un familiar, para 2015 5.517, mientras que para 2016 6.101. En segundo lugar se encuentra la pareja o la expareja y en tercer lugar los amigos. (P. 21).

Estas medidas de protección y atención, básicamente consisten en:

a. Garantizar la habitación y alimentación de la víctima a través del Sistema General de Seguridad Social en Salud. Las Empresas Promotoras de Salud y las Administradoras del Régimen Subsidiado en Salud, prestarán servicios de habitación y alimentación en las instituciones prestadoras de servicios de salud, o contratarán servicios de hotelería 
para tales fines; en todos los casos se incluirá el servicio de transporte de las víctimas y de sus hijos. Adicionalmente, contarán con sistemas de referencia y contrarreferencia para la atención de las víctimas, siempre garantizando la guarda de su vida, dignidad e integridad.

b. Cuando la víctima decida no permanecer en los servicios hoteleros disponibles, o estos no hayan sido contratados, se asignará un subsidio monetario mensual para la habitación y alimentación de la víctima, y de sus hijos, siempre y cuando se verifique que el mismo será utilizado para sufragar estos gastos en un lugar diferente al que habite el agresor. Así mismo, este subsidio estará condicionado a la asistencia a citas médicas, sicológicas o siquiátricas que requiera la víctima. En el régimen contributivo este subsidio será equivalente al monto de la cotización que haga la víctima al Sistema General de Seguridad Social en Salud, y para el régimen subsidiado será equivalente a un salario mínimo mensual vigente.

c. Las Empresas Promotoras de Salud y las Administradoras del Régimen Subsidiado serán las encargadas de la prestación de servicios de asistencia médica, sicológica y siquiátrica a las mujeres víctimas de violencia, a sus hijos e hijas. (Ley 1257, 2008, art. 19).

Lo anterior, desde el deber ser, constituye una verdadera herramienta de protección a los derechos de la mujer en condición de vulnerabilidad generada por la violencia. Dado que la ley no estableció mecanismos de financiación ni el procedimiento para acceder a las medidas de atención a la mujeres violentadas, el Gobierno expidió con posterioridad el Decreto 4796 del 2011', el Decreto 4799 del $2011^{9}$ y el Decreto 2734 del $2012^{10}$, por medio de los cuales reglamentó las medidas de protección a las mujeres víctimas de violencia así como también distribuyó competencias en materia de atención a las mujeres violentadas, entre los jueces, fiscalías y comisarías de familiar.

Estos decretos fijan los parámetros para suministrar las medidas de atención y hacen énfasis en la revisión de los casos en los que se solicitan estos mecanismos, con el fin de evitar que las mujeres que no sean víctimas de violencia se beneficien injustificadamente del programa creado expresamente para las mujeres violentadas. Dichos decretos fueron concebidos de conformidad con la declaratoria de excequibilidad de la Ley 1257 del 2008 que emitiera la Corte Constitucional, la cual consideró que el reglamento deberá contar con

8 "Por el cual se reglamentan parcialmente los artículos 8, 9, 13 y 19 de la Ley 1257 de 2008 y se dictan otras disposiciones". (Decreto 4796, 2011).

9 "Artículo 1. El presente decreto tiene por objeto reglamentar las Leyes 294 de 1996, 575 del 2000 y 1257 del 2008, en relación con las competencias de las Comisarías de Familia, la Fiscalía General de la Nación, los Juzgados Civiles y los Jueces de Control de Garantías, de manera que se garantice el efectivo acceso de las mujeres a los mecanismos y recursos que establece la ley para su protección, como instrumento para erradicar todas las formas de violencia contra ellas". (Decreto 4799, 2011).

10 "Artículo 1. Objeto y campo de aplicación. El presente decreto tiene por objeto establecer los criterios, condiciones y procedimiento para el otorgamiento de las medidas de atención definidas en el artículo 19 de la Ley 1257 del 2008, los cuales serán de obligatorio cumplimiento por parte de los diferentes actores del Sistema General de Seguridad Social en Salud -SGSSS y las autoridades competentes para ordenarlas en el marco de las responsabilidades que les fueron asignadas mediante la Ley 1257 del 2008 y sus Decretos Reglamentarios números 4796 y 4799 del 2011 y las normas que los modifiquen, adicionen o sustituyan". (Decreto 2734, 2012). 
[...] medidas encaminadas a evitar posibles abusos relacionados con reclamaciones presentadas por personas que pretendiendo obtener los beneficios y las medidas previstos en la ley, acudan ante las autoridades para reclamarlos sin haber sido víctimas de hechos constitutivos de violencia contra la mujer. (Sentencia C-776 del 2010).

Estas medidas de atención se conceden una vez agotadas las demás herramientas de protección a la mujer violentada, como la orden al agresor de abandonar el lugar de residencia de ella o la prohibición de acercarse a los lugares donde pernocta la víctima. Es decir, se deben ejercitar los medios de amparo femenino estipulados en el Código Penal y en la ley de violencia intrafamiliar antes de concluir que no logran salvaguardar los derechos por lo que es imperioso atender íntegramente a la víctima, brindándole techo, comida y transporte.

Concretamente, el Decreto 2734 del 2012 establece que las medidas de atención, consistentes en servicios temporales de habitación, alimentación y transporte que necesitan las mujeres víctimas de violencia con afectación física $y / 0$ psicológica, sus hijos e hijas, tienen aplicación bajo la recomendación de los profesionales de la salud, quienes deben fundamentar su juicio en la evaluación de la historia clínica.
Igualmente proceder cuando sea recomendada la reubicación de la víctima por parte de la Policía Nacional, una vez haya sido valorada la situación especial de riesgo.

El otorgamiento de las medias mencionadas corresponde a autoridades competentes, entre las que se encuentra la Comisaría de Familia; en ausencia de esta entidad, la decisión le corresponde al juez civil municipal o promiscuo municipal. Sin embargo, cuando sea la Fiscalía la que conozca hechos de violencia contra la mujer, será el juez de control de garantías el ente competente, ya sea por solicitud de esta o de la víctima. Así mismo, estas autoridades, antes de decretar las medidas de atención, deberán analizar minuciosamente el caso y atender con sumo cuidado las recomendaciones de la entidad de salud que lo atendió y de la Policía Nacional, de conformidad con lo expuesto anteriormente.

La Ley 1257 del 2008 creó dos procedimientos para acceder a las medidas, en consideración a la forma como se haya dado inicio a la atención de la violencia, haciendo la salvedad de que, en cualquier caso, la medida será transitoria, hasta por el término de 6 meses, prorrogable por otros 6 meses más, ya que se ordena con la finalidad de que la víctima pueda culminar su tratamiento $\mathrm{y}$ recuperarse. 
Tabla 1. Medidas de atención en favor de las víctimas de violencia intrafamiliar, de acuerdo al lugar de atención primaria según la Ley 1257 de 2008

Otorgamiento de medidas de atención cuando la víctima es atendida por el Sistema General de Seguridad Social en Salud o un régimen de salud especial o excepcional.
Otorgamiento de medidas de atención cuando la víctima denuncia el hecho de violencia ante la comisaría de familia o autoridad competente.

En la entidad de salud, la victima debe ser valorada para determinar si requiere tratamiento físico o psicológico, haciendo un resumen del evento dañoso y explicando la afectación de la mujer. Dentro de las 12 horas siguientes el hospital, IPS o similar, enviará dicha valoración a la autoridad competente, por lo general comisaría de familia o las demás en caso de que en dicho territorio no exista esta entidad.

La autoridad (comisaría) inicia el trámite de adopción de medidas de protección, de conformidad con artículo 4 de la Ley 294 de 1996. Constata que la víctima no esté incluida en ningún programa de protección y le consulta si está de acuerdo con las medidas, ya que no se podrá tomar determinaciones en contra de la voluntad de la mujer.

Dentro de las 12 horas siguientes a que la víctima haya aceptado las medidas, la autoridad debe solicitar a la Policía Nacional un informe de evaluación de riesgo, quien tendrá el término de 48 horas para hacer el estudio de riesgo y presentarse a la autoridad.

Con el informe de riesgo y aquel remitido por la entidad de salud que valoró a la mujer, la autoridad determinará si concede las medias de atención que consisten en alojamiento, alimentación y transporte de la víctima y sus hijos.

La autoridad debe proteger a la mujer decretando las medidas diferentes a la de atención, como ordenar el desalojo del agresor o prohibirle comunicación. Simultáneamente y con el fin de que la mujer sea valorada física y mentalmente debe remitir a la víctima a una clínica que tenga convenio con la entidad promotora de salud. Si no se encuentra afiliada al sistema de salud, será remitida a una empresa social del Estado.

Fuente: elaboración propia.

Otras medidas de atención establecidas por la ley mencionada son los subsidios monetarios, cuyo monto corresponde a un salario mínimo legal mensual vigente para aquellas mujeres que pertenezcan al régimen subsidiado; a las demás, el monto será aquel que corresponda a su cotización mensual. Su cancelación está a cargo de la Secretaría Departamental o Distrital. Procede en caso de que la víctima no acepte la habitación, alimentación y transporte para sus cuidados y recuperación, siempre y cuando se presente uno de los siguientes eventos:

1. En el departamento o distrito donde resida la mujer víctima no existan servicios de habitación contratados. 2.
En el municipio donde resida la mujer víctima no existan los servicios de habitación contratados y ella no pueda trasladarse del municipio por razones de trabajo. 3. Los cupos asignados en el departamento o distrito para servicios de habitación para las mujeres víctimas de violencia se hayan agotado. (Decreto 4796, 2011, art. 9).

Continuando con la exploración de las normas que protegen los derechos de las mujeres y que consagran instrumentos legales de defensa, es importante mencionar que el legislador, teniendo presentes los escenarios de violencia contra la mujer que muestran altos índices de trasgresión a sus derechos 
humanos, prosiguió con el diseño de herramientas jurídicas tendientes a brindarle mayor seguridad y protección.

Por ello en 2012 se promulgó la Ley 1542 que eliminó de los delitos de violencia intrafamiliar e inasistencia alimentaria el carácter de querellables y desistibles, tipificados en los artículos 229 y 233 del Código Penal, con el objeto de evitar que las denuncias por violencia sean conciliadas por la víctima, muchas veces, bajo la presión del agresor. Esta ley también permitió que la conducta punible contra la mujer sea investigada oficiosamente por la autoridad competente la cual, antes de la vigencia de la norma, debía esperar a la presentación de la denuncia por parte de la mujer para dar inicio al proceso penal. Esta ley fue motivada por los altos índices de desistimiento de las denuncias presentadas por maltrato intrafamiliar, indicios de que las mujeres eran constreñidas por sus agresores para que no continuaran con el proceso penal. Desafortunadamente, organizaciones feministas y de protección de los derechos de la mujer han informado que en algunos casos de violencia las mujeres se encuentran tan subyugadas que prefieren ocultar de la autoridad los hechos estigmatizantes, para que no sea iniciada una causa penal en contra de su pareja, ya que no pueden conciliar sus lesiones ni desistir de la persecución penal. En otros términos, el estado de vulnerabilidad de la víctima se agrava cuando tiene que pensar dos veces para denunciar a su verdugo, porque una vez se acude a la justicia no hay marcha atrás.

En 2015, después de analizar las cifras de los homicidios de mujeres en Colombia y detectar que la mera condición de ser mujer es el móvil del maltrato que en repetidas ocasiones culmina en asesinato, se expidió la Ley 1761 que endureció las penas para los delitos cometidos contra las mujeres en razón de su condición de género creando una nueva categoría de conducta punible a partir de la autonomía del homicidio en la persona de la mujer, denominada feminicidio. El delito comporta una pena hasta de 600 meses de prisión en la modalidad agravada.

En la norma se estipulan condiciones para la configuración del feminicidio, entre las cuales se encuentra que el agresor haya sostenido una relación con la víctima y haya perpetrado actos de violencia constantes, es decir, que exista prueba de maltrato o amenazas cometidas por el homicida en contra de la víctima, incluso sin que de ello obren denuncias.

Algunas de las circunstancias de agravación punitiva más relevantes están relacionadas con la edad de la víctima y su capacidad, estableciéndose como agravante que los sujetos pasivos sean mujeres mayores de 60 años o menores de 18 años o que se cometiere el delito en una mujer que se halle en situación de discapacidad.

Con base en el derecho a la igualdad y no discriminación también se consideró como agravante del delito el que este se cometa en razón de prejuicios relacionados con la condición étnica o la orientación sexual de la víctima.

\section{CRítica a la PROTECCIÓN Y DEFENSA DE LOS DERECHOS DE LA MUJER}

Luego de analizar el principal desarrollo normativo colombiano en favor de la protección y defensa de los derechos de la mujer, es necesario adelantar una discusión sobre 
la verdadera salvaguarda de la mujer violentada, en el entendido de que la expedición de una ley no conduce per se a garantizar los derechos de las personas ni a protegerles de abusos. Al respecto, Bobbio (1997) afirma que "el derecho real no es el que se encuentra enunciado en una Constitución, en un código, o en un cuerpo de leyes, sino aquel que los hombres efectivamente aplican en sus relaciones cotidianas" (p. 27).

Con relación a la efectiva participación de la mujer en los cargos del Estado, la norma ha dejado un sabor a desigualdad. Esta afirmación busca reflexionar sobre la idea de que las mujeres no tengan solo el $30 \%$ de participación en cargos público sino por lo menos la mitad, en criterio de equidad. No obstante, y en relación específica al cumplimiento de la ley de cuotas, se encuentra que en términos generales se ha logrado el objetivo y la mujer alcanzó a participar de los cargos nacionales y regionales en un porcentaje mayor al establecido por la ley.

En promedio el $59.7 \%$ de las entidades en todo el país cumplieron con el porcentaje establecido por la Ley 581 del 2000, al vincular mujeres en los cargos de alta dirección, con una participación de las mujeres del $30 \%$ o más. De igual forma, en promedio, el $46 \%$ de los cargos públicos provistos en la alta dirección son ocupados por mujeres. (Departamento Administrativo de la Función Pública, 2014, p. 46).

El empleo en la rama ejecutiva, en el maximo nivel decisorio estuvo ocupado en un $38 \%$ por mujeres; la rama judicial, en un $35 \%$; la rama legislativa, en un $70 \%$; los organos de control, en un $46 \%$; gobernaciones, en un $39 \%$; alcaldías, en un $41 \%$. (Departamento Administrativo de la Función Pública, 2015, p. 143).

Abordando la protección de la mujer rural y sin demeritar el programa "Mujer Rural" que brindó acompañamiento a las agricultoras, es necesario resaltar que aún no se crea en Colombia una política pública para atender los requerimientos de estas mujeres, especialmente en lo atinente al acceso a la tierra, base de su subsistencia. Desde la promulgación de la Ley 731 del 2002, el país está a la expectativa del compendio normativo acompañado de una juiciosa implementación, con el fin principal de empoderar a las mujeres rurales y proporcionarles herramientas de progreso y participación. El actual Plan de Desarrollo "Todos por un nuevo país", en su artículo $232^{11}$ plantea nuevamente el deber de elaborar dicha política pero no se observan aproximaciones a su construcción excepto un documento emanado de la Mesa de Incidencia, Política de las Mujeres Rurales Colombianas ${ }^{12}$, el cual expone los lineamientos de política pública para garantizar el acceso a tierras de las mujeres rurales, fijando unos principios orientadores que se deben tener en cuenta a la hora de redactar la política, a saber:

11 "Artículo 232. Política de mujer rural. Para la protección y garantía de los derechos de las mujeres rurales, el Gobierno nacional formulará una política pública integral de mujer rural de forma participativa, coordinada por el Ministerio de Agricultura y Desarrollo Rural y la Consejería Presidencial para la Equidad de la Mujer, que tendrá en cuenta el enfoque étnico, etario y territorial. Esta política pública estará orientada a superar las brechas urbano-rurales. En todo caso y con el fin de avanzar en la garantía de los derechos de las mujeres rurales, el Gobierno nacional implementará las medidas necesarias para dar pleno cumplimiento a lo preceptuado en la Ley 731 del 2002".

12 Esta entidad de carácter privado es un colectivo de organizaciones de mujeres campesinas, indígenas y afrocolombianas vinculadas a organizaciones de mujeres y mixtas del sector rural, de carácter nacional y regional para la incidencia política, la exigibilidad y el pleno ejercicio de derechos en pro de la mujer rural colombiana. 
a) Participación, b) autonomía, c) articulación institucional, d) sostenibilidad, e) integralidad, f) principio del uso social y agroecológico de la tierra, g) principio de equidad y prioridad del acceso a tierras, h) principio de integración, i) saberes ancestrales, j) transformación del contexto del territorio, $\mathrm{k}$ ) dimensión política. (Mesa de Incidencia. Política de las Mujeres Rurales Colombianas, 2015).

Sobre la protección de la mujer cabeza de familia, el panorama es más desalentador ya que después de la expedición de la Ley 82 de 1993 y su reforma por medio de la Ley 1232 del 2008, no se han establecido mecanismos eficaces para dar protección especial a la mujer que lleva la carga de su hogar, como programas especiales de educación, salud o vivienda, más allá de la estabilidad en el empleo (Ley 790 del 2002, art. 12 y Sentencia T-061 del 2006) y la prelación de aspirantes a cargos de empleos de carrera administrativa (Decreto Reglamentario 1227 del 2005, art. 7, parágrafo 2, numeral 2), entre otros tratamiento preferenciales que brinda la legislación doméstica. No existen propuestas concretas en el plan de desarrollo actual con fines de apoyo a las mujeres cabeza de hogar y los subsidios que actualmente otorga el programa Familias en Acción se entregan con base en unos criterios en los que se prioriza a estas mujeres, pero no se les otorgan beneficios exclusivos.

Ahora bien, luego de revisar el acceso efectivo de la mujer a cargos directivos de la administración pública y analizar la implementación de herramientas para proteger los derechos de la mujer rural y aquella que ejerce la jefatura del hogar, es hora de abrir la discusión sobre el cumplimiento de la norma que pretende erradicar la violencia contra la mujer y abordar especialmente lo relacionado con las disposiciones en materia de salud que se refieren a las medidas de atención. Siendo el mecanismo de financiación de estas medidas el primer elemento a observar, en consideración a que la Ley 1257 del 2008 indica que los recursos para implementar la atención a la mujer violentada serán proporcionados por el Sistema General de Seguridad Social en Salud, se impone el estudio del Decreto 4796 del 2011 el cual especifica las medidas que se concederán de acuerdo con los recursos disponibles, supeditando la garantía de los derechos humanos de las mujeres a la existencia de presupuesto para sufragar su atención.

El ejercicio de los derechos implica a menudo erogaciones estatales. Según Sunstein y Holmes (2011), la enunciación de garantías como meros postulados filosóficos o lo que podría llamarse, el reconocimiento de derechos, no genera en sí mismo obligaciones positivas por parte del Estado. Sin embargo, cuando se desarrolla el contenido de esos derechos y se establecen las medidas para su protección, siempre se requiere la disposición de recursos económicos para materializar los derechos en cuestión.

De conformidad con lo anterior, se estudió la Resolución 1895 del 2013, expedida cinco años después de la adopción de las medidas de atención en favor de las mujeres, cuya finalidad fue la de asignar recursos ${ }^{13}$ para dar cumplimiento a la norma y permitir el acceso de las víctimas a los medios de protección asistenciales, ya que es a las entidades promotoras de salud a las que les

13 Un total de $\$ 10962$ millones aportados entre el Fosyga y el Ministerio de la Protección Social. 
corresponde brindar la atención pero con cargo al sistema de salud, de modo que es el Gobierno el que, en última instancia, asume la carga presupuestal.

Después de verificar la apropiación presupuestal para el cumplimiento de la Ley 1257 del 2008 en lo que respecta al otorgamiento de las medidas de atención, consistentes en suministrar habitación, alimentación y transporte a la mujer víctima y a sus hijos, se aprecia que los resultados no dan cuenta de un escenario de garantía de los derechos de las mujeres, en la medida en que se han presentado obstáculos para el acceso a la mencionada protección.

En la concesión de las medidas de atención, las autoridades competentes se encuentran supeditadas al concepto de la entidad de salud que valoró a la víctima y a la evaluación de la Policía Nacional relacionada con el riesgo que corre la mujer en su lugar de residencia. Una vez certificada la viabilidad de la atención por parte de estas entidades, la autoridad competente (comisarías, jueces civiles municipales o promiscuos municipales y juez de control de garantías, según el caso) podrá ordenar la medida de atención en favor de la mujer y de sus hijos. Sin embargo, en la actualidad se observa una marcada vulneración de los derechos fundamentales de aquellos. En realidad, las entidades de salud suelen negar el acogimiento de la mujer y de sus hijos, argumentando dificultades técnicas y presupuestales o simplemente porque no aceptan su obligación de proporcionar las medidas de protección correspondientes.

En este sentido, la Sentencia T-434 del 2014 analizó un caso en el que la comisaría de familia decretó las medidas de atención y la entidad de salud se negó a otorgarlas, pese a que la normatividad al respecto obliga a las EPS a trasladar a la víctima al lugar de alojamiento, dentro de los tres días siguientes al decreto de las medidas. El argumento de la negativa, en primer lugar, se basó en que la entidad solo debe prestar a la víctima asistencia en salud, lo que no comporta hospedaje y demás emolumentos. En segundo lugar, explicó que unos meses después de la orden por parte de la comisaría se comunicó con la víctima para proporcionarle alojamiento, alimentación y transporte, pero esta junto a sus hijas, se encontraba fuera del municipio y no pretendía regresar al mismo.

A juicio de la corporación, la entidad promotora de salud vulneró los derechos de la víctima y de sus hijas al negarle la atención en los términos de lo ordenado por la comisaría de familia, y al demorarse en disponer del alojamiento y demás prestaciones. De hecho, el desplazamiento de la accionante se produjo principalmente por la ausencia de protección de sus derechos. En el caso examinado, la EPS debió proceder a gestionar el subsidio de que trata la Ley 1257 del 2008 en beneficio de la víctima para que, de manera temporal, sufrague los gastos de su manutención y la de sus hijas.

Esta decisión le recordó al Estado su obligación de adoptar medidas afirmativas con miras a dar cumplimiento a los postulados constitucionales y contribuir a la protección de la mujer, especialmente en casos de alta vulnerabilidad, en concordancia con el planteamiento de Acosta (2007) sobre la necesidad de "promover el cambio en la aplicación del derecho, al servicio de la justicia de género" (p. 57).

Respecto a la ley contra el feminicidio (también conocida como Ley Rosa Elvira Cely), 
con dos años de vigencia, es relativamente reciente y ya se han hecho sentir las sanciones penales a los victimarios, partiendo de la primera sentencia proferida por el órgano de cierre de la justicia penal, mediante la cual se condenó a un ciudadano a la pena de 23 años de prisión por asesinar a su compañera sentimental en medio de una historia de violencia y maltrato intrafamiliar.

La corporación enfatiza en que no todo el homicidio en la persona de una mujer puede considerarse feminicidio, toda vez que se debe analizar el escenario de ocurrencia para detectar el sometimiento y vulneración de la mujer a causa del comportamiento opresor de su agresor, en el que los celos extremos juegan un papel preponderante en la cosificación de la mujer. En ese sentido, la Corte puntualizó que:

Se causa la muerte a una mujer por el hecho de ser mujer, cuando el acto violento que la produce está determinado por la subordinación y discriminación de que es víctima, de lo cual resulta una situación de extrema vulnerabilidad. Este entorno de la violencia feminicida, que es expresión de una larga tradición de predominio del hombre sobre la mujer...No todo asesinato de una mujer es feminicidio y configura la causal 11 de agravación del artículo 104 del Código Penal. Se requiere, para constituir esa conducta, que la violencia que la cause esté asociada a la discriminación y dominación de que ella es objeto. (Corte Suprema de Justicia. Sentencia 41459 del 2015).

Se destacan las pocas condenas que se han proferido por el delito de feminicidio, las cuales son conocidas por las mujeres a través de los medios de comunicación que informan continuamente acerca de los procesos penales en varios departamentos del país por cuenta de esta conducta punible, sin embargo, todavía el grado de impunidad es muy alto debido a que el principal feminicida es el compañero sentimental y el tipo de violencia contra la mujer que termina con la extinción de su vida, es esencialmente intrafamiliar, sucede a menudo sin testigos, por lo que las mujeres temen denunciar a sus agresores y prefieren sufrir en silencio.

Analizar el fenómeno del homicidio a mujeres según el escenario del hecho nos pone frente a la paradoja de que tanto las vías públicas como las viviendas de las víctimas, sus espacios privados, son los dos lugares donde más son victimizadas las mujeres. Esto es válido para los años 2014, 2015 y 2016... El principal presunto agresor registrado es el agresor desconocido, seguido por la pareja o expareja. (Instituto de Medicina Legal y Ciencias Forenses, 2016, p. 9).

En el marco del maltrato y la opresión de los cuales son víctimas las mujeres, algunas agresiones físicas graves que podrían catalogarse en intentos de feminicidios son agravadas por la falta de atención por parte de las autoridades encargadas de protegerlas, que emprenden acciones contundentes ya cuando no hay nada que hacer y la violencia de género ha cobrado una nueva víctima. Lo anterior es corroborado por Bejarano (2014):

Las víctimas de violencia feminicida, ante la falta de apoyo y actuación efectiva de las autoridades, se ven obligadas a continuar en muchos casos viviendo 
con el agresor...; así pues, este tipo de violencia se cruza con la institucional. El tema de la justicia, su procuración por parte de las instituciones y de la justicia social en general, sigue siendo una asignatura pendiente para las mujeres que hacen frente y sobreviven a situaciones de violencia feminicida, así como para sus familias. (P. 35).

\section{Conclusiones}

De la revisión del desarrollo normativo de la protección de los derechos de la mujer en el país, se concluye que desde el deber ser se le han reconocido múltiples derechos a la mujer y se han diseñado mecanismos para su protección con fundamento en las disposiciones internacionales que han suscrito las autoridades para enfrentar la violencia y la marginación de las mujeres. Sin embargo, en lo que respecta a la efectiva garantía de sus derechos, persiste un escenario que dista de la protección demandada por ellas, por lo que:

Es necesario aplaudir algunos de los pasos dados hasta ahora, no se puede negar que a partir de 1991 el derecho constitucional es más cercano a las mujeres colombianas y a sus necesidades... resta un gran camino por recorrer. (Acosta, 2007, p. 49).

Desde los instrumentos internacionales en pro de la consagración de los derechos de la mujer, se instó a los Estados firmantes a que derogaran aquellas disposiciones legales que agravaban el estado de vulneración de la mujer, como el régimen matrimonial en el que se la asemejaba a otro hijo de familia y ni siquiera se le reconocían elementos esenciales de la personalidad jurídica (ciudadanía, capacidad y autonomía). En el mismo sentido, se convocó al mundo para que creara un robusto compendio jurídico que no solo generara respeto por los derechos de las mujeres, sino que les otorgara el verdadero ejercicio de los mismos e incluso prerrogativas, para que pudiesen alcanzar su proyecto vital.

Siguiendo las recomendaciones de organismos supranacionales, y en cumplimiento de los compromisos adquiridos, las ramas del poder público colombiano empezaron a pronunciarse con relación a los derechos de la mujer, especialmente en lo atinente a la eliminación de la discriminación y las medidas para protegerla de la violencia de género. Es así como desde el Congreso se han promulgado leyes que buscan equiparar los derechos de la mujer, así como prevenir y sancionar la violencia.

Se aprecia que el legislativo, incluso desde antes de la entrada en vigencia de la Constitución Política que hoy impera, empezó a facilitar el espacio político a la mujer, con el otorgamiento de la ciudadanía y el voto. Posteriormente, bajo el nuevo esquema constitucional, se dio inicio al debate de importantes iniciativas que culminaron en normas como la ley de cuotas, la de protección a la mujer cabeza de familia, la de fomento a la mujer rural, la de violencia intrafamiliar y la del feminicidio, entre otras.

Respecto de la protección de la mujer víctima de violencia, es necesario resaltar que en el plano normativo, la ley consagró importantes mecanismos para prevenir y sancionar los actos trasgresores de sus derechos, entre los que las medidas de atención se erigen como las más idóneas para proteger a 
la mujer víctima de violencia junto a sus hijos, puesto que establece que bajo ciertas circunstancias, puede acceder a hospedaje, alimentación y transporte para recuperarse del maltrato, lejos del agresor. De igual manera, el Gobierno nacional dispuso los recursos suficientes para aplicar la atención integral a la mujer con el fin de reintegrar a las EPS todos los valores pagados en desarrollo de las medidas. Sin embargo, en el país solo se registran pocos casos de aplicación de las medidas de atención.

De hecho, aunque mediante la Sentencia T-734 del 2014 la Corte Constitucional ordenó a una entidad de salud que diera cumplimiento a la ley, ya que la autoridad había ordenado atender a la mujer víctima de maltrato, la EPS se abstuvo de facilitarle el alojamiento y demás servicios a la víctima. Por ello, la mujer víctima de violencia de género tuvo que presentar una acción de tutela y esperar a que la alta corporación le concediera el derecho. Esto demuestra que la norma en sí misma no es suficiente para brindar protección a las mujeres víctimas de violencia y que actualmente están en medio de tal desprotección que amenaza sus propias vidas y las de sus hijos.

El Estado colombiano ha acatado lo establecido en normas y medidas internacionales para proteger a la mujer contra la discriminación y la violencia, y ha consagrado normas consecuentes con el derecho a la igualdad, que incluso ha generado espacios de participación a la mujer como los propiciados por la ley de cuotas, pero que los avances legislativos no se han traducido en la vigencia de los derechos de la mujer ya que, en la práctica, las víctimas deben superar grandes obstáculos para acceder a los mecanismos de protección de sus derechos.
Tal como lo plantea Wolfgans (2004), la garantía de los derechos fundamentales no solo comporta una obligación de respeto por parte del Estado; también es imperioso que la autoridad pública vele por el efectivo respeto de esos derechos para que todas las personas logren disfrutar de sus derechos en la cotidianidad.

De la revisión del compendio normativo existente en Colombia para proteger a la mujer, especialmente orientado a salvaguardarla de toda forma de violencia y a brindarle asistencia, y de la contrastación con las últimas cifras relacionadas con la violencia de género ${ }^{14}$, se concluye que los derechos de la mujer no se garantizan en debida forma y que aunque esta ha logrado espacios de participación, el ordenamiento jurídico ha sido insuficiente para transformar un comportamiento social que favorece la impunidad y perpetúa la violencia contra las mujeres.

Para efectos de facilitar la identificación de medidas de protección consagradas en la Ley 1257 de 2008, se elaboró la Tabla 1 que da cuenta de cada una de las medidas a adoptar según la atención primaria de la víctima, sea en el centro de salud o en el recinto de la autoridad competente como las comisarías de familia.

14 El Centro de Referencia Nacional sobre Violencia dio a conocer las cifras de violencia contra la mujer en lo que iba corrido del año 2015. Es así como señaló que entre enero y febrero del 2015, 126 mujeres fueron víctimas de homicidio y 2631 acudieron al Instituto para ser valoradas por un presunto delito sexual.

En el marco de la violencia intrafamiliar, 735 niñas y mujeres adolescentes fueron víctimas de violencia por parte de un familiar 0 cuidador y 6269 fueron víctimas de violencia de pareja. En cuanto a las mujeres mayores de 60 años, 119 de ellas fueron agredidas físicamente y 1482 fueron víctimas de violencia por parte de otros familiares. (Medicina Legal, 2015). 


\section{REFERENCIAS}

Acosta Alvarado, P. (2007). La protección de los derechos de las mujeres en la Constitución colombiana. Derecho del Estado, 49-60. DOI: https://doi.org/10.22370/ rcs.2015.66.208

Acto Legislativo 03 de 1954. Reformatorio de la Constitución nacional por el cual se otorga a la mujer el derecho activo y pasivo del sufragio. Agosto 27 de 1954. DOI: https://doi. org/10.5354/0719-5249.2003.42457

Aranda, E. (2005). Estudios sobre la Ley Integral contra la Violencia de Género. Madrid, España: Dykinson.

Bejarano, M. (2014). El feminicidio es solo la punta del iceberg. Región y Sociedad, (4), 13-44. DOI: https://doi.org/10.22198/ rys.2014.0.a85

Blanco, J. y Cárdenas, M. (2009). Las mujeres en la historia de Colombia, sus derechos, sus deberes. Prolegómenos. Derechos y Valores, XII (23), 143-158. DOI: https://doi.org/10.18359/prole.2501

Bobbio, N. (1997). Teoría general del derecho. Segunda reimpresión de la segunda edición. Bogotá D. C., Colombia: Temis.

Código Civil Colombiano (CCC). Ley 57 de 1887. Abril 15 de 1887 (Colombia).

Constitución Política de Colombia [Const.]. Julio 7 de 1991 (Colombia).

Corte Constitucional. (2010). Sentencia C-776, M. P. Jorge Iván Palacio, 29 de septiembre del 2010.

Corte Constitucional. (2014). Sentencia T-434, M. P. Luis Guillermo Guerrero Pérez, 3 de julio del 2014.

Corte Suprema de Justicia. (2015). Sala de Casación Penal. Proceso 41467, M. P. Patricia Salazar Cuellar, 4 de marzo del 2015.
Decreto 164. (2010)). Por el cual se crea una Comisión Intersectorial denominada "Mesa Interinstitucional para Erradicar la Violencia contra las Mujeres". Ministerio del Interior y de Justicia. 25 de enero del 2010. DOI: https://doi. org/10.22201/crim.unam000001c.2017. c41

Decreto 4463. (2011). Por medio del cual se reglamenta parcialmente la Ley 1257 del 2008. Ministerio del Trabajo. 25 de noviembre del 2011.

Decreto 4796. (2011). Por el cual se reglamentan parcialmente los artículos 8, 9, 13 y 19 de la Ley 1257 del 2008 y se dictan otras disposiciones. Ministerio de Salud y Protección Social. 20 de diciembre del 2011. DOI: https://doi. org/10.17230/nfp.12.86.10

Decreto 4798. (2011). Por el cual se reglamenta parcialmente la Ley 1257 de 2008, "Por la cual se dictan normas de sensibilización, prevención y sanción de formas de violencia y discriminación contra las mujeres, se reforman los Códigos Penal, de Procedimiento Penal, la Ley 294 de 1996 y se dictan otras disposiciones". Ministerio de Educación Nacional. 20 de diciembre 20 del 2011.

Decreto 4799. (2011). Por el cual se reglamentan parcialmente las Leyes 294 de 1996, 575 del 2000 y 1257 del 2008. Ministerio del Interior y de Justicia. 20 de diciembre del 2011. DOI: https://doi. org/10.15332/s1900-0448.2017.0047.05

Decreto 2733. (2012). Por medio del cual se reglamenta el artículo 23 de la Ley 1257 del 2008. Ministerio de Hacienda y Crédito Público. 27 de diciembre del 2012. DOI: https://doi.org/10.17230/ nfp.12.86.9 
Decreto 2734. (2012). Por el cual se reglamentan las medidas de atención a las mujeres víctimas de violencia. Ministerio de Salud y Protección Social. 27 de diciembre del 2012. DOI: https://doi.org/10.2307/j.ctt1k232vs.11

Departamento Administrativo de la Función Pública. (2014). Informe sobre la participación femenina en el desempeño de cargos directivos de la administración pública colombiana. Bogotá D. C., Colombia. DOI: https://doi.org/10.11117/2236 1766.54.10.2369

Departamento Administrativo de la Función Pública. (2015). Informe de cumplimiento de la ley de cuotas en la administración pública colombiana. Bogotá D. C., Colombia. DOI: https://doi.org/10.18042/cepc/ rap.206.09

Equidad de la Mujer. Presidencia de la Republica. (12 de agosto del 2016). El entorno familiar, un lugar de riesgo para las mujeres colombianas. DOI: https://doi. org/10.4185/rlcs-2016-1103

Facio, A. y Fries, L. (1999). Género y derecho. Santiago de Chile, Chile: Lom Ediciones Gómez Molina, P. (2015). Régimen patrimonial del matrimonio: contexto histórico que rodeó la promulgación de la Ley 28 de 1932. Estudios Socio-Jurídicos, 41-76. DOI: https://doi.org/10.12804/ esj17.01.2014.02

Hernández, R., Fernandez, C. y Baptista, P. (2008). Metodologia de la investigación. México D. F., México: Edamsa Impresiones.

Instituto Nacional de Medicina Legal y Ciencias Forenses. (2015). Información estadística de violencia contra la mujer. Bogotá D. C., Colombia: Centro de Referencia Nacional sobre Violencia. DOI: https://doi.org/10.22209/msiu.n3a33
Instituto Nacional de Medicina Legal y Ciencias Forenses. (2016). Violencia de género en Colombia. Análisis comparativo de las cifras de los años 2014, 2015 y 2016. Violencia de Género en Colombia. Boletín Epidemiológico. Bogotá D. C., Colombia. DOI: https://doi.org/10.22209/msiu.n3a33

Ley 82. (1993). Por la cual se expiden normas para apoyar de manera especial a la mujer cabeza de familia. Diario Oficial n. 41101 de la República de Colombia. Bogotá D. C., Colombia, 3 de noviembre de 1993.

Ley 581. (2000). Por la cual se reglamenta la adecuada y efectiva participación de la mujer en los niveles decisorios de las diferentes ramas y órganos del poder público, de conformidad con los artículos 13, 40 y 43 de la Constitución. Diario Oficial n. ${ }^{\circ} 4026$ de la República de Colombia. Bogotá D. C., Colombia, 31 de mayo del 2000. DOI: https://doi. org/10.2307/j.ctv8bt39d.11

Ley 731. (2002). Por la cual se dictan normas para favorecer a las mujeres rurales. Diario Oficial n. 44678 de la República de Colombia. Bogotá D. C., Colombia, 14 de enero del 2002.

Ley 823. (2003). Por la cual se dictan normas sobre igualdad de oportunidades para las mujeres. Diario Oficial n. ${ }^{\circ} 45245$ de la República de Colombia. Bogotá D. C., Colombia, 7 de julio del 2003.

Ley 1232. (2008). Por la cual se modifica la Ley 82 de 1993, Ley Mujer Cabeza de Familia y se dictan otras disposiciones. Diario Oficial n.o 47053 de la República de Colombia. Bogotá D. C., Colombia, 17 de julio del 2008. DOI: https://doi. org/10.17230/nfp.12.86.10

Ley 1257. (2008). Por la cual se dictan normas de sensibilización, prevención 
y sanción de formas de violencia y discriminación contra las mujeres, se reforman los Códigos Penal, de Procedimiento Penal, la Ley 294 de 1996 y se dictan otras disposiciones. Diario Oficial n.o 47193 de la República de Colombia. Bogotá D. C., Colombia, 4 de diciembre del 2008. DOI: https://doi. org/10.17230/nfp.12.86.10

Ley 1542. (2012). Por la cual se reforma el artículo 74 de la Ley 906 del 2004, Código de Procedimiento Penal. Diario Oficial n.․ 48482 de la República de Colombia. Bogotá D. C., Colombia, 5 de julio del 2012. DOI: https://doi.org/10.17230/ nfp.12.86.10

Ley 1761. (2015). Por la cual se crea el tipo Penal de Feminicidio como delito autónomo y se dictan otras disposiciones. Diario Oficial n.․․ 49565 de la República de Colombia. Bogotá D. C., Colombia, 6 de julio del 2015. DOI: https://doi. org/10.17230/nfp.12.86.7

Ley 1753. (2015). Por la cual se expide el Plan Nacional de Desarrollo 2014-2018 "Todos por un nuevo país". Diario Oficial n. ${ }^{\circ} 49538$ de la República de Colombia. Bogotá D. C., Colombia, 9 de junio del 2015.

Mesa de Incidencia. Política de las Mujeres Rurales Colombianas. (2015). La tierra, más que un sueño. Lineamientos de la política pública para el acceso a tierras de las mujeres rurales. DOI: https://doi. org/10.19053/01218530.n33.2018.8142
Ministerio de Agricultura. (6 de marzo del 2015). Vamos a darle oportunidades y a saldar la deuda que tenemos con la mujer rural. Bogotá D. C., Colombia: Minagricultura. DOI: https://doi.org/10.2307/j. ctv6hp3sq.11

Montoya Ruiz, A. (2009). Mujeres y ciudadanía plena, miradas a la historia jurídica colombiana. Opinión Jurídica, 8(16), 137-148.

Procuraduría General de la Nación. (2004). Género, justicia y derecho. Bogotá D. C., Colombia: Universidad Externado de Colombia.

Resolución 1895. (2013). Por los cual se asignan recursos para la financiación de las medidas de atención a las mujeres víctimas de violencia, del que trata los literales a y b del artículo 19 de la Ley 1257 del 2008, para la vigencia fiscal del 2013. Ministerio de Salud y Protección Social. 31 de mayo del 2013. DOI: https://doi. org/10.1017/s0250569x0002015x

Rodríguez-Escobar, G. y Rodríguez-Escobar, M. (2014). Violencia sexual contra las mujeres en el conflicto armado colombiano: un desconocimiento de su dignidad. Revista Colombiana de Bioética, 9(2), 73-84. DOI: https://doi. org/10.18270/rcb.v9i2.712

Sunstein, C. y Holmes, S. (2011). El costo de los derechos. Buenos Aires, Argentina: Siglo Veintiuno Editores.

Wolfgans Sarlet, I. (2004). La eficacia de los derechos fundamentales. Sao Pablo, Brasil: Livraria Advogado. 